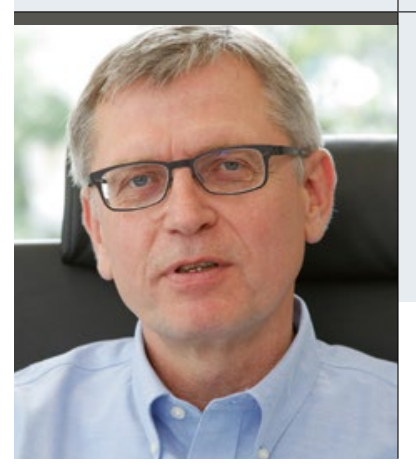

"Mit dieser Serie wollen wir eine Lücke in unserem

psychopharmakologischen Wissen schließen."

Prof. Dr. med. Hans-Peter Volz

Krankenhaus für Psychiatrie, Psychotherapie und Psychosomatische Medizin

Schloss Werneck

\title{
Was Sie schon immer über Off-Label wissen wollten ...
}

n dieser Ausgabe von DNP Der Neurologe \& Psychiater beginnt eine neue Serie mit Beiträgen zum Off-Label-Gebrauch von Psychopharmaka. Den ersten Artikel über den Einsatz von Medikamenten, die keine Zulassung zur Therapie von Angststörungen besitzen, schrieb Kollege Professor Ion-George Anghelescu, Facharzt für Psychiatrie und Psychotherapie, Chefarzt an der Privat-Nerven-Klinik Dr. Kurt Fontheim in Liebenburg. Weitere werden folgen: In der nächsten Ausgabe geht es um Schizophrenie, dann um Demenz und um die bipolare Störung. In der September-Ausgabe werden abschließend die bei Off-Label-Gebrauch besonders zu beachtenden rechtlichen Aspekte behandelt.

\section{Off-Label - was heißt das für Psychopharmaka?}

Off-Label bedeutet, lax übersetzt, außerhalb der zugelassenen Indikation. Hier muss man zunächst innehalten und sich fragen, wie ein Medikament für eine bestimmte Indikation überhaupt eine Zulassung erhält. Im Bereich der Psychopharmaka gibt es da zwei große Klassen: 1. Ältere Präparate, die häufig eine syndromgestützte Zulassung beispielsweise für Angstsymptome (unterschiedlicher Ätiologie) vor vielen Jahren erhalten haben und nun breit, also sowohl bei der generalisierten Angststörung als auch bei der Panikstörung eingesetzt werden können, und 2. neuere Medikamente, die auf aktuelle Diagnosemanuale gestützt (in der Regel kommt hier das amerikanische DSM-System zum Einsatz) eine Zulassung streng für eine oder mehrere klar umrissene Diagnosen erhalten. Insbesondere bei dieser zweiten Klasse von Medikamenten ergibt sich dann die Frage, warum gerade nur eine oder zwei Indikationen zugelassen wurden, und nicht auch andere. Anghelescu nennt in seinem überaus lesenswerten Artikel in dieser Ausgabe (Seite $34 \mathrm{ff}$ ) die Hauptgründe:
_Die Durchführung eines breiten klinischen Entwicklungsprogramms in weiteren, zusätzlichen Indikationen sprengt die finanziellen und/oder logistischen Möglichkeiten des Patentinhabers (in der Regel eine pharmazeutische Firma).

_Ein Entwicklungsprogramm wurde durchgeführt, allerdings konnten die notwendigen Bedingungen für eine Zulassung (z. B. mindestens zwei positive placebokontrollierte Studien) nicht erreicht werden.

_Eine Zulassung ist zwar erfolgt, im Rahmen der Bewertung eines Zusatznutzens durch den Gemeinsamen Bundesausschuss wurde aber kein Zusatznutzen festgestellt, was häufig dazu führt, dass das entsprechende Medikament dann nicht mehr vertrieben wird (nachdem es bereits zugelassen und für einen kurzen Zeitraum auf dem Markt war).

_ Im Rahmen strategischer Überlegungen des patenthaltenden pharmazeutischen Unternehmens wurde aktiv gegen die Entwicklung einer neuen Indikation entschieden (z. B. um den Markterfolg der Substanz in einer bereits zugelassenen Hauptindikation nicht zu gefährden).

\section{Höhere Anforderungen ...}

Es gibt sicherlich noch mehr Gründe, warum ein Medikament keine Zulassung in einer bestimmten Indikation hat, das Ergebnis ist aber immer gleich: Möchte man nun doch dieses Medikament einsetzen, hat man höhere Anforderungen zu berücksichtigen. Schon bei der bestimmungsmäßigen Anwendung eines Pharmakons muss der Patient über Vorund Nachteile intensiv aufgeklärt werden, hierzu gehört auch das Aufzeigen von therapeutischen Alternativen. Möchte man ein Medikament in einer Off-Label-Indikation einsetzen, sind hier die Anforderungen ungleich höher: Es muss eindeutig begründet werden, warum die Wahl nicht auf ein Me- 


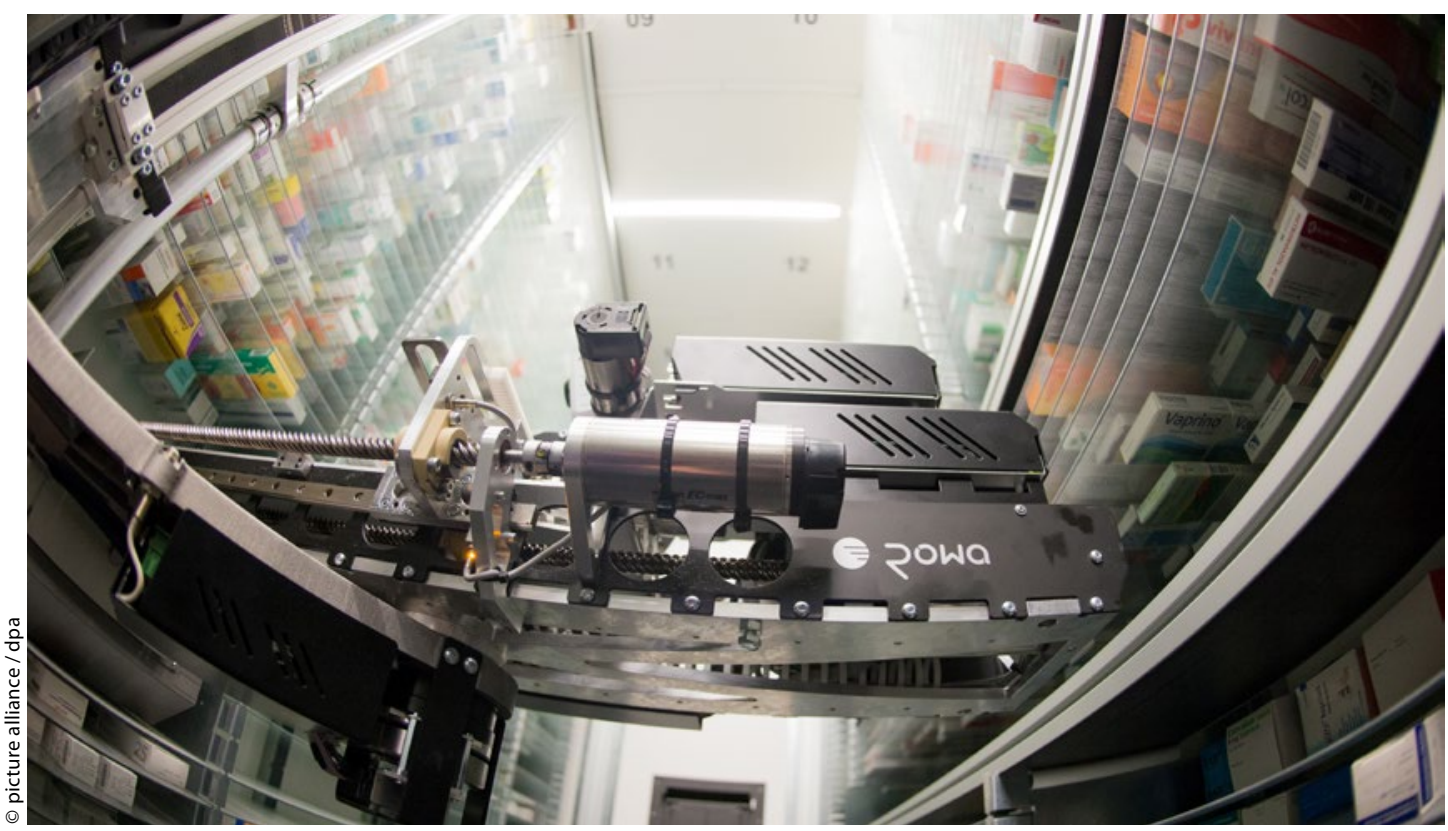

Wie lange ein zugelassenes Medikament auf dem Markt verfügbar ist, hängt manchmal davon ab, ob ein Zusatznutzen festgestellt wird.

dikament fällt, das für die gegebene Indikation eine Zulassung besitzt, und die Nutzen-Risiko-Abwägung muss besonders gründlich erfolgen. Vor allem muss der Patient nach einer solchen Aufklärung in der auch sehr deutlich verbalisiert werden muss, dass ein für die Indikation nicht zugelassenes Medikament zum Einsatz kommt -, einverstanden sein, und auch dieses Einverständnis sollte der Arzt gründlich dokumentieren.

\section{... aber auch zusätzliche Möglichkeiten}

Dann eröffnet sich allerdings ein breites Feld zusätzlicher therapeutischer Möglichkeiten, und welcher therapeutisch tätige Arzt wollte das nicht kennen und nutzen. Allerdings ist unser Wissen über solche Möglichkeiten in den letzten Jahren doch deutlich zurückgegangen, da zum Beispiel die Etablierung von Leitlinien, aber auch die verstärkte Aufmerksamkeit der Kostenerstatter, dazu geführt hat, dass sehr viel stärker als vor einigen Jahren auf den bestimmungsgemäßen Gebrauch der Medikamente, also auch auf die Verordnung nur für zugelassene Indikationen, geachtet wird. Insofern empfehle ich Ihnen unsere Serie zu den Off-Label-Möglichkeiten, die in dieser Ausgabe mit dem Beitrag von Kollege Anghelescu beginnt, - wird doch so hoffentlich eine sich immer breiter darstellende Lücke in unserem psychopharmakologischen Wissen geschlossen. Diese kleine Serie ist auch ein Experiment: Wir sind sehr daran interessiert zu erfahren, ob diese Beiträge für die Kollegen relevant sind und ob Kollegen überhaupt daran interessiert sind, neue Wege in der psychopharmalogischen Therapie zu gehen, trotz eines deutlich erhöhten Aufklärungsaufwands und auch haftungsrechtlicher Hürden. Falls ja, wird es für den aufgeschlossenen Kollegen auch möglich sein, den einen oder anderen schwer zu behandelnden Fall einer Symptombesserung zuzuführen, also das primäre Ziel eines jeden ärztlichen Handelns, Symptome zu lindern oder zu beheben, besser zu erreichen.

In diesem Sinne wünsche ich Ihnen eine spannende und interessante Lektüre.

\section{Mit freundlichen kollegialen Grüßen,} Ihr

\section{Hans-Peter Volz}

Prof. Dr. med. H.-P. Volz

Krankenhaus für Psychiatrie, Psychotherapie und Psychosomatische Medizin Schloss Werneck Balthasar-Neumann-Platz 1, 97440 Werneck E-Mail: hans-peter.volz@kh-schloss-werneck.de 